\section{André Torre et Maryline Filippi (sous la direction de) \\ Proximités et changements socio-économiques dans les mondes ruraux \\ Paris, INRA Éditions, 2005, 332 p.}

On le devine bien, cet ouvrage cadre tout à fait avec le précédent; d'ailleurs, l'une des responsables, Maryline Filippi, est chercheure (on dit chercheuse en France) à l'INRA de Toulouse. Pour sa part, André Torre, familier à certains collaborateurs d'O\&T, est directeur de recherche à l'UMR SAD-APT (un truc sérieux...) à Paris. Faisant appel à pas moins de 26 collaborateurs, cet ouvrage se veut on ne peut mieux pluridisciplinaire (économie, géographie, droit, psychologie, sociologie) en ayant pour objectif de montrer comment l'analyse de la proximité facilite la compréhension des mutations qui affectent les activités agricoles et agroalimentaires et des espaces ruraux en prenant en compte leurs répercussions sur l’organisation des territoires.

Les deux responsables de l'ouvrage débutent leur chapitre sur les mutations en œuvre par une allusion à l'imaginaire qu'a pu inspirer le monde rural : «Considérés comme les garants des traditions, capables d'assurer la pérennité des lieux de mémoire et la permanence des relations sociales et productives, l'espace rural et les activités agricoles occupent une place particulière dans l'imaginaire national ». Peut-on trouver un meilleur lien avec l'ouvrage qui précède ? (Voir les dernières lignes de mon commentaire.) Oui, l'imaginaire québécois ne déroge pas à ce qui s'observe chez nos cousins. Toujours selon Torre et Filippi, on assiste de nos jours à un processus original de construction des territoires. Il s'agit d'un mouvement de masse qui s'explique à la fois par les politiques publiques mises de l'avant et par une volonté des populations locales de se retrouver et de s'identifier autour de représentations communes opérant dans le cadre d'une construction sociale de nature collective. On croirait lire une étude sur notre politique nationale de la ruralité... Faisant toujours allusion aux mutations en cours, ces deux auteurs évoquent l'évolution de la relation ville-campagne et la " renaissance » des espaces ruraux. On se rapporte ici à la perte d'influence des activités agricoles au profit des activités de transformation et de services. Dès lors qu'on ne considère plus uniquement l'activité agricole, on parle en conséquence, en citant Kaiser, de la naissance de nouvelles campagnes. C'est ce à quoi s'intéresse, d'un océan à l'autre, depuis plusieurs années la Fondation canadienne de revitalisation rurale et le nouveau Réseau national de recherche rurale (traduction boiteuse mais officielle de National Rural Research Network) dont font partie certains collaborateurs d'O\& T.

La section sur la gouvernance territoriale en intéressera plus d'un. Il y est fait allusion à une gouvernance exercée au sein de dispositifs territoriaux ayant pour objet la mise en place de collectifs d'acteurs locaux autour d'un problème et la médiation entre ces acteurs et le monde matériel. Ces dispositifs territoriaux, entre autres choses, peuvent se positionner à l'interface entre incitation institutionnelle et initiative locale dans la construction de territoires de projets. Mais puisqu'il faut évoquer la proximité, il devient nécessaire de se rapporter aux travaux réalisés depuis le milieu des années 1990 sur l'économie de la proximité. Il est ici question de proximité géographique facile à concevoir et de proximité organisationnelle presque aussi facile à imaginer. Disons, comme le précisent les auteurs, que cette dernière n'est pas d'essence géographique mais relationnelle. Elle se rapporte à la capacité qu'offre une organisation de faire interagir ses membres. Plus loin, un autre auteur parlera de proximité institutionnelle sans vraiment faire la distinction avec celle dont il est question ici. Enfin, tout le monde comprend qu'il est abondamment question ici de réseautage sous une forme ou une autre.

Ce livre est fait partiellement de contributions déjà publiées. Comme la probabilité que les lecteurs d'O\&T en aient déjà pris connaissance s'avère presque nulle, nous y verrons ici avant tout un gage de la qualité de ces contributions. C'est le cas notamment des chapitres suivants : Dynamiques des firmes et politiques de développement régional et local; Mutations de la grande distribution et évolution du commerce; Les proximités, la ville et le rural; Réorganisation dans la coopération agricole : proximités et solidarités territoriale.

Les quelque quinze chapitres étant répartis en quatre parties, c'est la troisième, intitulée Gouvernance des espaces ruraux, qui a retenu particulièrement mon attention. Deux types d'espaces ruraux se trouvent mis en évidence : les périurbains, définis par l’intensité des migrations alternantes et caractérisés par de fortes relations à la ville, et les «traditionnels » ou « autonomes », moins liés à la ville, bien au vert (les chan- 
ceux!). Deux auteurs proposent trois grands groupes de localisation et de besoins de proximité qui permettent de fonder une définition de trois catégories d'espace : les villes, les espaces ruraux périurbains et les espaces éloignés bien verdoyants. Ne reste plus qu'à voir comment ces derniers peuvent se sentir moins éloignés grâce aux technologies de l'information et de communication. C'est l'objet de l'ouvrage suivant.

\section{Guide TIC des petites et moyennes collectivités : à l'usage des élus, cadres et fonctionnaires territoriaux Paris, FICOME, 2004, 124 p.}

La Fédération interprofessionnelle de la communication d'entreprise (FICOME) souhaite, par ce petit ouvrage, accroître l'efficacité des administrations tout en assurant un accès permanent aux services publics pour les citoyens et les entreprises bien conscients de leurs responsabilités dans le développement économique. Fibre optique ? ASDL ? WiFi ? Courants en ligne ? Voilà autant de questions dont un début de réponse se trouve dans un premier chapitre portant sur l'accès au haut débit. Pour que le lecteur québécois s'y retrouve avec tous ces sigles et expressions d'oùtre-Atlantique, voire d'outre-Manche, en annexe un lexique "numérique » permet d'en découdre avec les ASP, ASDL, BLR, CPL, HFC, IP-PBX, LAN, MAN, PABX et autres UMTS, ou encore WAN, WAP. Quant à WiFi, nous savions tous que ça réfère à Wireless Fidelity que les Montréalais connaissent grâce à leurs cafés Secund Cup.

Pour les rédacteurs de ce guide, le Top 4 des usages pour une petite collectivité se présente ainsi :

a) E-administration : il s'agit de généraliser l'usage du courriel (e-mail dans le texte).

b) Augmenter la qualité de la vie par les points d'accès à l'Internet. Ici, notre beau et grand pays n'a pas de leçons à recevoir.

c) Rendre le territoire plus compétitif par l'accès au haut débit pour les entreprises. Ici, le Canada n'est malheureusement pas le "plus meilleur » pays du monde...

d) Renforcer la sécurité des biens et des personnes en situation de crise. On évoque les inondations, les tremblements de terre, les incendies de forêt... Il est vrai que, si les infortunés de Nouvelle-Orléans avaient été mieux branchés, on aurait pu plus facilement prendre connaissance de leurs malheurs... Mais il ne s'agit pas d'une petite ni même moyenne collectivité.

On revient à nos moutons en considérant la section la plus intéressante pour nos élus et ceux qui gravitent tout autour : le développement économique et l'emploi. On évoque surtout ici les conditions d'accueil de nouvelles entreprises en se rapportant à la technologie qui permet le partage d'équipements télécoms et le partage sécurisé de ressources personnalisées entre différents groupes d'abonnés ou d'entreprises. Ici, on parle d'équipement $\mathrm{PBX}$ (si nécessaire; prière de consulter le lexique en annexe). En fait, comme on le pense bien, tout ce beau monde doit travailler la main dans la main. C'est que démontre la prochaine recension.

\section{"L’inter-communalité » Revue Regards sur l'actualité Paris, La Documentation Française, $n^{\circ} 314$, octobre 2005, 91 p.}

Ce numéro de la revue Regards sur l'actualité porte essentiellement sur un sujet qui fait couler passablement d'encre en France depuis des années. Quand on sait qu'il existe en Hexagone pas moins de 36565 municipalités, dites communes, soit $40 \%$ de ce que compte l'Union européenne à vingt-cinq, ça en fait des maires et des conseillers municipaux, comme dirait le père Ovide. De là l'importance de faire en sorte que tout ce beau monde puisse se parler. Pour ce faire, on a créé exprès un nouveau sigle : EPCI (Établissement public de coopération intercommunale). Quand on connaît l'importance de l'esprit de clocher qui a toujours caractérisé tous les Clochemerles des descendants de nos ancêtres les Gaulois, l’expression « révolution intercommunale » n'est pas trop forte pour souligner le changement apparu dans les années 1990. En effet, à travers les CIDA (oui, vous avez bien lu : Chartes intercommunales de développement et d'aménagement), on apprenait à travailler ensemble plutôt que de toujours se prendre au chignon. Ainsi, petit train va loin, au $1^{\mathrm{er}}$ janvier la fille aînée de l’Église comptait pas moins de 2525 EPCI ressemblant $88 \%$ des communes et $82 \%$ de la population. Félicitations 